Within the past few months two excellent summaries $(1,2)$ of the state of our knowledge of the weak interactions have been presented. Correspondingly, we will not attempt a comprehensive review but instead concentrate this discussion on the status of $\mathrm{CP}$ violation; the question of the neutral currents, and the weak equivalence principle.

\title{
The Parameters
}

The phenomenon of $\mathrm{CP}$ violation has been definitely observed only in the neutral $\mathrm{K}$ meson system. It is a tiny effect but nevertheless, many of the parameters which characterize the violation have been determined with some crispness. It is not surprising that this phenomenon has yet to be seen in other systems since the neutral $K$ system is so extraordinarily sensitive, several orders of magnitude more sensitive than any other system known. The searches for evidence of $\mathrm{CP}$ violation in other systems have been made in the fond hope that the small effect in the neutral $\mathrm{K}$ system was, in fact, a massive effect in some other channel. Unfortunately, they have been false hopes. The status of the searches for $C P$ or $T$ violation in other systems will be summarized later.

To date, $10 \mathrm{CP}$ violating parameters in the neutral $\mathrm{K}$ system have been measured. However, some of these have yet to be measured to the accuracy necessary to make the result relevant to the immediate questions. These 


\section{DISCLAIMER}

This report was prepared as an account of work sponsored by an agency of the United States Government. Neither the United States Government nor any agency Thereof, nor any of their employees, makes any warranty, express or implied, or assumes any legal liability or responsibility for the accuracy, completeness, or usefulness of any information, apparatus, product, or process disclosed, or represents that its use would not infringe privately owned rights. Reference herein to any specific commercial product, process, or service by trade name, trademark, manufacturer, or otherwise does not necessarily constitute or imply its endorsement, recommendation, or favoring by the United States Government or any agency thereof. The views and opinions of authors expressed herein do not necessarily state or reflect those of the United States Government or any agency thereof. 


\section{DISCLAIMER}

Portions of this document may be illegible in electronic image products. Images are produced from the best available original document. 
parameters are (we assume CPT invariance throughout),

$$
\begin{aligned}
& \eta_{+-}=\frac{\operatorname{Ampl}\left(K_{L} \rightarrow \pi^{+} \pi^{-}\right)}{\operatorname{Ampl}\left(K_{S} \rightarrow \pi^{+} \pi^{-}\right)} \quad \text { phase and magnitude } \\
& \eta_{\infty}=\frac{\operatorname{Ampl}\left(K_{L} \rightarrow 2 \pi^{\circ}\right)}{A m p l\left(K_{S} \rightarrow 2 \pi^{\circ}\right)} \quad \text { phase and magnitude } \\
& \operatorname{Re} \epsilon=\operatorname{Re}\left[\frac{1}{3}\left(2 \eta_{+-}+\eta_{\infty}\right)\right] \\
& \operatorname{Imag} \mathbf{x}^{*}=\operatorname{Imag} \frac{\operatorname{Ampl}\left(\mathrm{K}^{\circ} \rightarrow \pi^{+} \mu^{-} \nu\right)}{\operatorname{Ampl}\left(\mathrm{K}^{\circ} \rightarrow \pi^{-} \mu^{+} \nu\right)} \\
& \text { Imag } x=\operatorname{Imag} \frac{\operatorname{Ampl}\left(K^{\circ} \rightarrow \pi^{+} e^{-} \nu\right)}{\operatorname{Ampl}\left(K^{\circ} \rightarrow \pi^{-} e^{+} \nu\right)} \\
& \eta_{+-0}=\frac{\operatorname{Ampl}\left(K_{S} \rightarrow \pi^{+} \pi^{-} \pi^{o}\right)}{\operatorname{Ampl}\left(K_{L} \rightarrow \pi^{+} \pi^{-} \pi^{0}\right)} \quad \text { phase and magnitude. }
\end{aligned}
$$

Imag $\xi$ = Imag part of form factor ratio in $\mathrm{K}_{\mu 3}$ decay

There remain several other parameters, e.g.

$$
\eta_{000}=\frac{\operatorname{Ampl}\left(K_{S} \rightarrow 3 \pi^{\circ}\right)}{\operatorname{Ampl}\left(K_{L}-3 \pi^{\circ}\right)},
$$

that have not been measured at all. As has been recognized for a long time, a convincing demonstration of a difference between $\eta_{+-}$and $\eta_{00}$ would be highly informative as far as diagnosing the ultimate source of CP violation. If, on the other hand, $\eta_{+_{-}}=\eta_{\text {oo }}$ within the experimental errors, a wide range of possibilities remain. (3) 
Eta Plus Minus

About the magnitude there is little question. Since the days of the initial observation the value has hovered around the current best value, viz.

$$
\left|\eta_{+-}\right|=1.95 \pm 0.03 \times 10^{-3}
$$

The phase, as one might expect, is trickier to measure and its value has seen wilder oscillations. Three years ago a judicious summary ${ }^{(4)}$ of the data available yielded arg $\eta_{+_{-}}=\varphi_{+-}=59^{\circ} \pm 6^{\circ}$. Now several independent measurements using different techniques lead to a different number but one in which one can have, hopefully, a fair amount of confidence. As examples of completely different techniques $I$ will discuss the last two reported measurements. The first is that of the Chicago group working at Argonne. (5) They used so-called "vacuum regeneration" i.e. the fact that with CP violation the time structure in the $\pi^{+} \pi^{-}$rate after production of a $\mathrm{K}^{\circ}$ should go as

$$
\begin{aligned}
I\left(\pi^{+} \pi^{-}\right) & \alpha \mid e^{-(i \delta+I / 2) t / 2 \tau_{S}+\left.\eta_{+-}\right|^{2}} \\
& =e^{-t / \tau_{S}}+\left|\eta_{+-}\right|^{2}+2\left|\eta_{+-}\right| e^{-t / 2 \tau} \cos \left(\delta\left(\frac{t}{\tau_{S}}\right)+\varphi_{+-}\right)
\end{aligned}
$$

Interference is clearly seen in the region where the $K_{S}^{\circ}$ and $K_{L}^{0}$ amplitudes are roughly the same (i.e. where $e^{-t / 2 \tau_{S}} \cong\left|\eta_{+-}\right|$or where $t \cong 12 \tau_{S}$ ) and from it the phase $\left(\varphi_{+-}+\delta t / \tau_{S}\right)$ is determined. In the sensitive region, 
$8 t / \tau_{S} \cong 300^{\circ}$ so the technique is extremely sensitive to the $K_{L}^{0}-K_{S}^{0}$ mass difference, $\delta=\left(m_{L}-m_{S}\right) \tau_{S} / \hbar$. The Chicago result is

$$
\varphi_{+-}=42.4^{\circ}+310.0^{\circ}\left(1-0.538 \times 10^{10} \frac{T_{S}}{\delta}\right) \pm 4.0^{\circ} \text {. }
$$

Fortunately, in the past year, three separate measurements of the mass difference have been made, each to about $1 \%$, and they all agree!: $(6,7,8)$ The combined result is

$$
\frac{\delta}{\tau_{S}}=0.5390 \pm .0035 \times 10^{10} \mathrm{sec}^{-1}
$$

or

$$
\frac{\delta}{\tau_{S}}=858 \mathrm{Mc} \quad \text { (L band!) }
$$

When doing interferometry with neutral K's it is convenient to remember that this mass difference leads to a phase shift between $K_{S}^{0}$ and $K_{L}^{0}$ amplitudes at the same energy of $26.4^{\circ}$ per $\mathrm{K}_{\mathrm{S}}^{\circ}$ mean life.

This value for the mass difference leads to

$$
\varphi_{+-}=43.0^{\circ} \pm 5.0^{\circ}
$$

A group from Princeton (9) working at the A.G.S. have measured $\varphi_{+-}$ in one self-contained experiment using a technique which is completely orthogonal to that described above. The interference effects between the $K_{L} \rightarrow \pi^{+} \pi^{-}$and the $K_{S} \rightarrow \pi^{+} \pi^{-}$amplitudes when the $K_{S}^{\circ} s$ have been produced by coherent regeneration have been a powerful tool. The intensity of the forward going decays to $\pi^{+} \pi^{-}$as a function of time $T$ measured in units of the $k_{1}$ mean life is 


$$
\begin{aligned}
I\left(\pi^{+} \pi^{-}\right) & =|\rho|^{2} e^{-\tau}+2\left|\eta_{+-}\right||\rho| e^{-\tau / 2} \cos \left(\delta \tau+\varphi_{\rho}+\varphi_{+-}\right) \\
& +\left|\eta_{+-}\right|^{2} .
\end{aligned}
$$

Turning our attention to the 3-body leptonic decays and utilizing the $\Delta S=\Delta Q$ rule whereby $K^{0} \rightarrow \pi^{-} e^{+} \nu$ and $\bar{K}^{\circ} \rightarrow \pi^{+} e^{-} \nu$, the $K_{e 3}$ charge asymmetry behind a regenerator resulting from the decay of forward going $K^{\prime} s$ has a time dependence given by:

$$
A=\frac{N\left(e^{+} \pi^{-} \nu\right)-N\left(e^{-} \pi^{+} \bar{\nu}\right)}{N\left(e^{+} \pi^{-} \nu\right)+N\left(e^{-} \pi^{+} \bar{\nu}\right)}=2|\rho| e^{-\tau / 2} \cos \left(\varphi_{\rho}-\delta \tau\right)
$$

where $\rho$ is the $K_{S}^{0}$ amplitude immediately behind the regenerator (with the 9 1/8" thick $\mathrm{Cu}$ regenerator used in this experiment $|\rho| \sim 0.1)$. The main idea of the measurement was to compare, in the same apparatus with the data taken concurrently, the structure in proper time in the $\mathrm{K}_{\mathrm{e}}$ asymmetry and the $\pi^{+} \pi^{-}$rate. The relative phase of the two structures yields $\varphi_{+-}$. The neatness of the above procedure is somewhat muddied by the fact that the $K_{e 3}$ decays originating from diffractively scattered $K^{\prime} s$ exhibit an asymmetry almost opposite in sign to the coherently scattered. Furthermore the 3-body decay does not permit a clean separation of the decays originating from coherently scattered $\mathrm{K}$ 's from those diffractively scattered. In fact, summing the scattering to all orders leads to an effective regeneration amplitude

$$
\rho_{\text {eff }}=\rho\left(1-2 \frac{\sigma_{D}}{\sigma_{T}} \zeta \cos \varphi^{\prime} e^{-i \varphi^{\prime}}\right) .
$$


where $\zeta$ is the relative apparatus efficiency for detecting the diffractively scattered $K_{L}^{0}$ 's and in $\varphi^{\prime}=\arg [-i(f(0)-\bar{f}(0))]$ we have collected our ignorance of the scattering which we were originally trying to avoid. The $\sigma_{\mathrm{D}} / \sigma_{\mathrm{T}}$ is the ratio of the diffraction to the total cross sections. For the record we note that Eq. (3) is a simplification of the complete expression.

$$
A(\tau)=\frac{2\left(1-|x|^{2}\right)\left[|\rho| e^{-\tau / 2} \cos \left(\varphi_{\rho}-\delta \tau\right)+\operatorname{Re} \epsilon\right]}{|1-x|^{2}-4|\rho| e^{-\tau / 2} \operatorname{Im} x \sin \left(\varphi_{\rho}-d \tau\right)}
$$

where $\mathrm{x}=[(\Delta S=-\Delta Q)$ amplitude $/(\Delta S=\Delta Q)$ amplitude $]$. While it appears that the unavoidable presence of the diffractively scattered $\mathrm{K}^{\circ}$ 's substantially dilutes the crispness of the original experimental plan, the data have been parameterized in such a way as to permit separation into varying degrees of enrichment of the forward scattered, coherent, component and thereby make possible an extrapolation to a zero diffractively scattered component. The separation parameter used was

$$
\Delta=p_{\nu}\left(c_{\cdot} m_{*}\right)-p_{\nu L}
$$

Figure 1 illustrates the effectiveness of the parameter $\Delta$ in separating the coherent from the incoherently scattered K's. Figure 2 shows that the fit to the asymmetry as a function of particular $\Delta$ bands. The $K$ momentum accepted by this apparatus ranged from 1.2 to $4 \mathrm{BeV}$ with a weighted mean of $2.6 \mathrm{BeV}$.

Whereas the asymmetry measurement is self-normalizing and the result 
is independent of the detection efficiency, the measurement of the interference in the $2 \pi$ rate is not intrinsically self-normalizing. However, in this experiment, the data were made self-normalizing by recording them with the regenerator in two different positions. When the regenerator was in the "near position" to the volume in which the $2 \pi$ decays were measured $\left(\left|p e^{-(i \delta+I / 2) \tau}\right| \gg\left|\eta_{+-}\right|\right.$throughout the pertinent volume $)$little interference was present and the data served mainly to calibrate the proper time dependence of the detection efficiency. In the "far" position, a proper time $\tau_{0}$ upstream, $\left|p e^{-(i \delta+1 / 2)\left(\tau+\tau_{0}\right)} \sim \eta_{+_{-}}\right|$, the interference effects are maximized. The ratio of the proper time dependences at the far and near positions displays the interference effects independent of detection efficiency. The resulting phase comparison between the $\mathrm{K}_{\mathrm{e}}$ asymmetry and $K_{\pi 2}$ interference was done as a function of momentum. The difference, assuming it to be independent of momentum, is

$$
\varphi_{+-}=36.2^{\circ} \pm 6.0^{\circ},
$$

about one $\sigma$ away from the "vacuum regeneration" result. The weighted mean of this result and that from "vacuum regeneration is $40.3^{\circ} \pm 3.9^{\circ}$. If one includes in the averaging all of the more recent data ${ }^{(10)}$ one gets a somewhat higher number, viz.

$$
\varphi_{+-}=41.8^{\circ} \pm 3.0^{\circ}
$$

At this point it is appropriate to observe that the "natural" phase is

$$
\arg (1 / i \delta+1 / 2)=43.0^{\circ}+0.4^{\circ}
$$




\section{$-8-$}

We have assumed throughout that $m_{i}>m_{S}$. This has recently been reconfirmed by a Carnegie Tech., BNL Case group (II) who conclude that $\mathrm{m}_{L}>$ $\mathrm{m}_{\mathrm{S}}$ is $\sim 10^{5}$ times more likely than the reverse.

\section{Eta Nought Nought}

The history of the attempts to measure $\eta_{\text {oo }}$ has been turbulent for good reason. The rare decay of $K_{L} \rightarrow 2 \pi^{\circ}$ competes with a dominant mode $K_{L} \rightarrow 3 \pi^{\circ}$. The photons are difficult to measure because among the $6 \gamma^{\prime} s$ in the $3 \pi^{\circ}$ mode, 2 are very likely to be going backward in the c.m. system. They, correspondingly, have very little energy in the laboratory, and are not easily detected. The other 4 photons carry the full energy of the $K$ with only transverse components as signatures. In Fig. 3 we have collected the various results. $(12,13,14,15,16,17,18,19,20)$ since the rate is almost always the measured quantity we have plotted $\left|\eta_{00}\right|^{2}$. of special note is the result from the liquid Xenon chamber, Barmin et al., (15) a truly monumental bubble chamber experiment, and the most recent result from CERN, Darriulat et al., reported at the Amsterdam conference. (19) Table I tabulates the data.

Clearly the results are not consistent -- and there is no apparent reason for rejecting any one measurement. A weighted average of all of the results leads to

$$
\begin{aligned}
\left|\eta_{00}\right|^{2} & =4.3 \pm 0.4 \times 10^{-6} \text { internal errors } \\
& =4.3 \pm 0.7 \times 10^{-6}=\text { external errors. }
\end{aligned}
$$

We subsequently use the number with the larger error.

$$
\text { Chollet, et al., (17) have also studied the interference pattern in the }
$$


$-9-$

$2 \pi^{\circ}$ rate behind the regenerator and arrive at $43.0^{\circ} \pm 19.0^{\circ}$ for the phase, a result, they emphasize, which is largely independent of the magnitude.

We have then, summarizing all the data

$$
\begin{aligned}
& \eta_{00}=2.08 \pm 0.16 \times 10^{-3} \text { e } \mathrm{e}^{143.0^{\circ} \pm 19.0^{\circ}} \\
& \pi_{-}=1.95 \pm 0.03 \times 10^{-3} \mathrm{e}^{141.8^{\circ} \pm 3.0^{\circ}} .
\end{aligned}
$$

Except for some lingering reservations about $\left|\eta_{o 0}\right|$ one can feel fairly comPortable about the four numbers.

Status of the Wu-Yang Triangle

We assume CPT and re-express the 4 parameters determined above in terms of those associated with the mass decay matrix, ${ }^{(21)}$ viz.

$$
\begin{array}{rl}
c=\frac{2}{3} \eta_{+-}+\frac{1}{3} \eta_{00} & c=\frac{p-q}{p+q} \\
c^{\prime}=\frac{1}{3}\left(\eta_{+-}-\eta_{00}\right) & \varepsilon^{\prime}=\frac{1 I m A_{2}}{2 \sqrt{A_{0}}} e^{i\left(\delta_{2}-\delta_{0}\right)}
\end{array}
$$

where

$$
\begin{aligned}
& K_{L}^{0}=\left[I / \sqrt{p^{2}+q^{2}}\right]\left[p K^{0}+q \bar{K}^{\circ}\right] \\
& K_{S}^{0}=\left[I / \sqrt{p^{2}+q^{2}}\right]\left[p K^{0}-q^{\circ} \bar{K}^{\circ}\right] .
\end{aligned}
$$

Inserting the above information on $\eta_{00}$ and $\eta_{+-}$we have,

$$
\operatorname{Re} \varepsilon^{\prime}=-.03+.18
$$




$$
\because \quad \text { In } \epsilon^{\prime}=-.03+.16
$$

with the errors correlated so that $\varepsilon^{\prime}$ is either in the first or third quadrant.

In short, $\left|\varepsilon^{\prime}\right|$ is not more than $\sim 20 \%$ of $\left|\eta_{+-}\right|$. But tighter limits can be placed on arg $\varepsilon^{\prime}$ from our knowledge of the $\pi \pi$ phase shifts -- deriveable $e^{(22)}$ from the $\mathrm{K}^{+} \rightarrow \pi^{+} \pi^{\circ}$ decay rate and the ratio

$$
R=\frac{\Gamma\left(K_{S}^{0} \rightarrow \pi^{+} \pi^{-}\right)}{\Gamma\left(K_{S}^{0} \rightarrow 2 \pi^{\circ}\right)} .
$$

A recently obtained $(23,24)$ average for $R$ yields $R=2.22 \pm .03$, the departure from 2 being the contribution of the $T=2$ amplitude given by

$$
R=.986\left(2+6 \sqrt{2} \operatorname{Re} A_{2}^{0} / A_{0} \cos \left(\delta_{2} \rightarrow \delta_{0}\right)\right)+\Delta_{e m}
$$

where $\Delta_{\mathrm{em}}$, the electromagnetic correction, has been estimated to be $\sim .006 \pm$ .04. (25) One can, with good justification (invoking, e.g, the Cabibbo hypothesis), ignore possible $|\Delta I|=5 / 2$ transitions, whereupon $A_{2}^{0} / A_{0}=2 / 3 A_{2}^{+} / A_{0}$. From $\mathrm{K}^{+} \rightarrow \pi^{+} \pi^{0}$ decay we have $\mathrm{A}_{2}^{+} / \mathrm{A}_{0}=.054$. We obtain then, $\cos \left(\delta_{2}-\delta_{0}\right)=$ $.63 \pm .16$ and

$$
\left|\delta_{2}-\delta_{0}\right|=51.0^{\circ} \pm 12.0^{\circ}
$$

The analysis of multipion production data has led to values of $\delta_{0}$ with this same magnitude and in the first quadrant. With $\delta_{2}$ small we use this information and take accordingly. 


$$
8_{2}-\sigma_{0}=-51.0^{\circ} \pm 12.0^{\circ}
$$

whereupon: the $\arg \epsilon^{\prime}=39.0^{\circ} \pm 12.0^{\circ}$. Since arg $\epsilon^{\prime}$ is close to $\arg \eta_{+-}$the Wu Yang triangle tends toward a Wu Yang line. Inserting this new constraint we have

$$
c^{\prime}=-.04 \pm 0.06 e^{139.0^{0} \pm 12.0^{0}} \times 10^{-3}
$$

and

$$
\varepsilon=1.99 \pm 0.073^{143.0^{\circ} \pm 3.0^{\circ}} \times 10^{-3}
$$

\section{Charge Asymmetry}

Because with $C P$ violation

$$
\mathrm{K}_{\mathrm{L}}^{0} \cong\left(\mathrm{K}^{0}+\overline{\mathrm{K}}^{0}\right)+\varepsilon\left(\mathrm{K}^{0}-\overline{\mathrm{K}}^{0}\right)
$$

one has the $K_{L}^{0}$ decaying to $\pi l \nu$ in a charge asymmetric way,

$$
A=\frac{N\left(l^{+}\right)-N\left(l^{-}\right)}{N\left(l^{+}\right)+N\left(l^{-}\right)}=\frac{1-|x|^{2}}{|1-x|^{2}} 2 \operatorname{Re} \epsilon
$$

where $\mathrm{x}$ is the ratio of the $\Delta S=-\Delta Q$ amplitude to the $\Delta S=\Delta Q$ amplitude.

We should keep an open mind and allow for the possibility that $\mathrm{x}$ is different for the $K_{e 3}$ and $K_{\mu 3}$ decay mode's and consider the data on the charge asymmetry separately. However, the $K_{\mu 3}$ data is sufficiently few that this distinction is not justified and we Iump everything together. We emphasize the small effect one is trying to measure -- with $x=0$, $2 \operatorname{Re} \epsilon=A \sim 2.9 \times 10^{-3}$.

For the $\mathrm{K}_{\mu 3}$ mode one measurement exists. Curiously, the measurement of the asymmetry in the $\mathrm{K}_{\mathrm{e}}$ mode has been attacked more vigorously -- sta- 
tistically speaking. Three older results and a preliminary result from a new experiment, just being analyzed, are available and shown in Table II. Again the dispersion in the results is rather large. The weighted

mean is

$$
\begin{aligned}
A & =2.88 \pm 0.20 \times 10^{-3} & & \text { internal error } \\
& =2.88 \pm 0.27 \times 10^{-3} & & \text { external error } .
\end{aligned}
$$

This number is to be compared with the predicted asymmetry using as input the value of $\varepsilon$ determined from $\eta_{4-}$ and $\eta_{00^{\circ}}$ With $x=0$ we have

$$
A=2 \operatorname{Re} \varepsilon=2.90 \pm 0.17 \times 10^{-3} \text { (predicted). }
$$

The difference between the predicted and the measured value is $(0.02 \pm 0.35)$ $\times 10^{-3}$. Turning the question around and asking for the permissable $\Delta S=\Delta Q$ violation consistent with this data, we obtain $\operatorname{Re} x=.00 \pm .06$ and an insignificant sensitivity to Im $x$.

A departure from the $\Delta S=\Delta Q$ rule was first reported at Aix-en-Provence ten years ago -- slightly more than a $2 \sigma$ effect. In the meantime many experiments have been done with progressively smaller errors but always about $2 \sigma$ from zero. Weighted averages of all the data available always demonstrated an effect. Now the result from a single high statistics experiment has become available from a CERN-Orsay-Vienna group which shows no violation of the rule. The current results, reported at the recent Amsterdam conference, $(31,32)$ are

$$
\text { For } K_{e 3}\left\{\begin{array}{l}
\operatorname{Re} x=.05-.025 \\
\operatorname{Im} x=-.01 \pm .02
\end{array}\right.
$$




$$
\text { For } \mathrm{K}_{\mu 3} \quad\left\{\begin{array}{l}
\operatorname{Re} \mathrm{x}^{\prime}=-.09 \pm 0.10 \\
\operatorname{Im} \mathrm{x}^{\prime}=0.1 \pm 0.15
\end{array}\right.
$$

Values of $x$ permitted by the errors assigned to these results can change the predicted asymmetry for the $\mathrm{K}_{\mathrm{e} 3}$ decay by $\sim 20 \%$.

\section{General Remarks}

As seen above, on the basis of the present information, $\varepsilon^{\prime}$ is small (if not zero) compared to $\varepsilon$. Therefore the CP violation is largely in the mass-decay matrix as opposed to the $T=2$ decay amplitude $\varepsilon^{\prime}$.

We recall

$$
\varepsilon=\frac{p-q}{p+q}=\frac{p^{2}-q^{2}}{(p+q)^{2}}=\frac{\left(\Gamma_{12}-\Gamma_{21}\right)+i\left(M_{12}-M_{21}\right)}{2(i \delta+1 / 2)}
$$

where all rates and masses are measured in units of $\Gamma_{s}$. With $M_{12}=M_{R}+$ i $M_{i}=M_{21}^{*}$ and $\Gamma_{12}=\Gamma_{21}^{\dagger}$,

$$
\epsilon=\frac{i \operatorname{Im} \Gamma_{12}+M_{i}}{i \delta+I / 2}
$$

Clearly, the measured phase of $\varepsilon$ requires $M_{i} \gg \operatorname{Im} \Gamma_{12^{*}}$. As was originally pointed out by Wu and Yang in 1964, ${ }^{(21)}$ if one allows for a maximum CP violation in the $3 \pi$ and $K_{\ell 3}$ channels, one still cannot account from measured 
rates for all of $\varepsilon--$ one needs some $M_{i}$. It is of interest to update their observation on the basis of the latest data. The Imag $\Gamma_{12}$ term contributes to $\epsilon$ at right angles to the natural phase, viz., $\arg (1 /(i \delta+1 / 2))=43.0^{\circ}$ and, therefore, the measured phase of $\varepsilon$ provides constraints on the magnitude of Imag $\Gamma_{12}$. Independently, from the measured decay rates, the maximum contributions to Imag $\Gamma_{12}$ can be obtained and these are listed in Table III. The calculation for the $3 \pi^{\circ}$ channel assumes the same isotopic spin states are involved as for $\pi^{+} \pi^{-} \pi^{\circ}$ channel. (1.) Barring some gross misbehavior of $\eta_{0}$, the sum of the right hand column in Table III, taking into account the errors, can contribute only a tiny fraction of $|\epsilon|$ to the $\mathrm{CP}$ violation. This is consistent with the conclusion one draws from the measured phase of $\epsilon$, viz., the $1 / 20$ radian error in arg $\varepsilon$ would permit a contribution of $2 / 20 \times 10^{-3}=0.1 \times 10^{-3}$ to Imag $\Gamma_{12}$, comparable to the larger components in the table.

We must conclude that the major contribution to the CP violation is from the imaginary parts of the off-diagonal elements of the mass matrix. Once one puts the effect in the mass matrix it is correspondingly more inaccessible experimentally, since it involves the exploration of the off-themass-shell CP violating effects.

Where does the ultimate source of $\mathrm{CP}$ violation reside? As we noted in the beginning, if $\eta_{+_{-}} \neq \eta_{00}$, our diagnosis of the source would be simplified. However, since they are not distinctly different we must continue to explore nearly all possibilities. A spectrum of these possibilities was summarized by Wolfenstein ${ }^{(33)}$ a few years ago and we reproduce from his paper a listing 
of various models for $C P$ violation and their predicted manifestations. This listing is given in Table IV. W We have updated the data in the row devoted to experimental results.

A pemisal of the table reveals very few models that can be eliminated, especially when it is recognized that the model prediction, $\eta_{+-} \neq \eta_{00}$ is an exact statement, small inequalities are permitted by the models and, indeed, in many cases are most likely. More specifically, any model of $\mathrm{CP}$-nonconservation which violates the $\Delta I=1 / 2$ rule to the same degree it is violated in $\mathrm{CP}$ conserving reactions would not lead to a large enough difference between $\eta_{00}$ and $\eta_{t_{-}}$to be seen in present experiments. One sees the $\Delta I=5 / 2$ model definitely eliminated and the e.m. violation put in an uncomfortable but not completely untenable position by the information about the current limit on the electric dipole moment of the neutron. That is all.

The Table emphasizes another important point. Nearly all of the accessory experiments that have been performed to seek other evidences of $\mathrm{CP}$ violation are still too insensitive by one, two, and sometimes three orders of magnitude. The experimental physicists have their work well delineated in the future.

Table IV also demonstrates that the superweak model perhaps enjoys an unjustified popularity since there is clearly no more evidence in its favor than there is for many other models.

\section{Strangeness Changing Neutral Currents}

The classical test for the existence of strangeness changing neutral 
currents has been the search for the decay mode $K_{L}^{0} \rightarrow \mu^{+} \mu^{-}$. Until recently the information on this decay mode had come as a by-product from other experiments, most recently those experiments that have been devoted to studying the $2 \pi$ decay of the $K_{L}^{\circ}$. Three years ago a group at Berkeley initiated an experiment specifically targeted toward pushing the neutral current limit down to the point where the $2 \mu$ decay mode was expected from straightforward, presumably well understood, electromagnetic processes. In the absence of electromagnetic effects one can also have $2 \mu$ 's from 2 nd order weak processes but this is well below the expected electromagnetic threshold. It was always expected that the electromagnetic effects would lead to a $\mathrm{K}_{\mathrm{L}} \rightarrow 2 \mu$ branching ratio of the order of $10^{-8}$ and this was a targeted number. More precisely, on the basis of the measured $\mathrm{BR}\left(\mathrm{K}_{\mathrm{L}}^{\mathrm{O}} \rightarrow 2 \gamma\right)=5.2 \times 10^{-4}$, the lower limit for $\mathrm{K}^{\circ} \rightarrow 2 \mu$ has been calculated to be $\mathrm{BR}\left(\mathrm{K}_{\mathrm{L}}^{\circ} \rightarrow 2 \mu\right) \gtrsim 6 \times 10^{-9}$. (34) The result of the Berkeley Bevatron (35) experiment was one dubious event from which they compute a $\mathrm{BR}\left(\mathrm{K}_{\mathrm{L}} \rightarrow 2 \mu\right)=6.8 \times 10^{-10}$. The probability of getting $I$ event (even a good event) or less when you are entitled to at least 10 is

$$
\text { Prob } \leq 11 \mathrm{e}^{-10} \cong 10^{-3} / 2
$$

Since the theoretical estimate is a lower bound a reasonable probability is much less than this. So it is clear we are dealing with an absolutely extraordinary bad mu of statistics, or a deceptive theoretical estimate, or some exciting new physics. The experiment has no perceptible faults. It has been meticuiously performed. With respect to the theoretical estimate for the EM rate, it should be noted that in the case of eta decay a similar 
estimate appears to work, i.e.

$$
\begin{array}{rlrl}
\frac{\Gamma\left(\eta \rightarrow \mu^{+} \mu^{-}\right)}{\Gamma(\eta \rightarrow Y \gamma)} & \geq 1.1 \times 10^{-5} & & \text { predicted } \\
(36)
\end{array}
$$

In short, there is a problem interpreting this experimental result within the framework of our present knowledge. Christ and Lee ${ }^{(38)}$ and M. K Gaillard (39) have suggested a way out involving CP violation. They invoke $K_{1}^{0}$ and $K_{2}^{\circ}$ decay amplitudes to $2 \mu$ which destructively interfere. We note that the $\mu^{-\mu^{+}}$system in a ${ }^{3} P_{0}$ state has $\mathrm{CP}=+1$ and in a ${ }^{1} S_{0}$ state has $C P=-1$. We recall

$$
\begin{aligned}
K_{L}^{0} & =\left(K^{0}+\bar{K}^{0}\right)+\epsilon\left(K^{0}-\bar{K}^{0}\right) \\
& =K_{2}^{0}+\epsilon K_{1}^{0} .
\end{aligned}
$$

For the amplitudes for $K_{1}^{0}$ and $K_{2}^{0}$ decay to interfere they must decay to the same state, either ${ }^{3} P_{0}$ or ${ }^{I_{S}}$ and therefore either the $K_{2}^{0}$ or the $\mathrm{K}_{1}^{\mathrm{O}}$ decay must violate $\mathrm{CP}$.

If the $K_{2}^{0}$ rate to $2 \mu$ 's is near the unitarity limit then the CP violating rate for $K_{1}^{0}$ and $2 \mu$ 's would have to be $\sim\left(\frac{1}{\varepsilon}\right)^{2}$ times as large. The Christ-Lee estimate is

$$
\mathrm{BR}=\frac{\Gamma\left(\mathrm{K}_{\mathrm{S}} \rightarrow 2 \mu\right)}{\Gamma\left(\mathrm{K}_{\mathrm{S}} \rightarrow \mathrm{all}\right)}>5 \times 10^{-7}
$$


The current limit on $\mathrm{K}_{\mathrm{S}} \rightarrow 2 \mu$ comes from Hyams et al. (40) and is within a factor of 10 of addressing the current question.

Their result is

$$
\frac{\Gamma\left(\mathrm{K}_{\mathrm{S}} \rightarrow 2 \mu\right)}{\Gamma\left(\mathrm{K}_{\mathrm{S}} \rightarrow \mathrm{all}\right)} \leq 7.3 \times 10^{-6} 90 \% \text { confidence. }
$$

Needless to say, these developments have initiated great interest in studying $\mathrm{K}_{\mathrm{S}}^{\circ} \rightarrow 2 \mu$ as well as $\mathrm{K}_{\mathrm{S}}^{\circ} \rightarrow 2 \gamma$. Three different experiments devoted to the latter decay have been reported. Cline et al., (4l) Gaillard et al., (42) and Nauenberg et al., ${ }^{(43)}$ each have limits of $\sim 2 \times 10^{-3}$. Combined, the limit becomes $\lesssim 10^{-3}$.

The Weak Equivalence Principle

Ten years ago M. L. Good ${ }^{(44)}$ observed that the neutral $K$ meson system provided a sensitive test of the Equivalence Principle in Relativity. He r'served that if the $\mathrm{K}^{\circ}$ and $\overline{\mathrm{K}}^{\circ}$ experienced gravitational forces of opposite sign they would be mixed so quickly after the production of a $K^{\circ}$ or $\bar{K}^{\circ}$, the $K_{L}^{O}$ would not exist. He established limits by using the (apparent) absence of $K_{L}^{O}$ decay to 2 pions and concluded that the $K^{\circ}$ and $\bar{K}^{\circ}$ had the same gravitational mass to within about $.7 \times 10^{-7}$ if one were dealing with an isolated earth, about .5 $\times 10^{-8}$ for an isolated solar system, and $\sim 10^{-10}$ if one considered an isolated galaxy. The equal gravitational mass of particle and antiparticle is a manifestation of the so-called weak equivalence principle of general relativity $(45)$ as contrasted to the "strong" equivalence of gravitational and inertial mass.

It is of considerable interest to ask what new limits can be set on the 
weak equivalence principle using all the new and rather refined data on the neutral $\mathrm{K}$ system. (46)

On the surface of an isolated earth, radius $R$, the gravitational potential energies ${ }^{(47)}$ of the $K^{\circ}$ and $\bar{K}^{\circ}$ at rest are

$$
\mathrm{V}_{\mathrm{K}^{\circ}}=\mathrm{M}_{\mathrm{K}^{g R}}(I+x)
$$

and

$$
\mathrm{V}_{\overline{\mathrm{K}}} \mathrm{O}=\mathrm{M}_{\mathrm{K}} \mathrm{gR}(I-x)
$$

where $g$ is the usual gravitational acceleration and where $x$, the fractional difference between the particle and antiparticle gravitational mass, parameterizes the violation of the weak equivalence principle.

We note that

$$
\frac{\mathrm{V}_{\mathrm{K}} \mathrm{O}}{\mathrm{M}_{\mathrm{K}} \mathrm{C}^{2}}=\frac{\mathrm{gR}}{\mathrm{C}^{2}} \cong 7 \times 10^{-10}
$$

or

$$
\mathrm{V}_{\mathrm{KO}} \cong 0.35 \mathrm{eV}
$$

The potentials appear in the diagonal elements of the mass-decay matrix and lead to a relative amplitude for $\mathrm{K}_{\mathrm{L}}^{\circ} \rightarrow 2 \pi$ decay compared to $\mathrm{K}_{\mathrm{S}}^{\circ} \rightarrow 2 \pi$ of

$$
\Delta=\frac{i x V_{K^{\circ}} T_{S}}{(i \delta+1 / 2) \not h}
$$

where now, since $|\Delta| \ll\left|\eta_{+-}\right|=1.9 \times 10^{-3}$ and $h / \tau_{s}=8 \times 10^{-6} \mathrm{ev}$, one can see the enormous sensitivity.

Two effects serve to distinguish the effect of a failure of the weak equivalence principle from a violation of CP. First, $\Delta$ is $90^{\circ}$ out of phase 
with the measured $\eta_{+-}$or $\epsilon$. Second, we expect the potential $V_{K^{\circ}}$ to transform from the rest system to the moving system with a

$$
\gamma^{2}=\left(\frac{E_{K}}{M_{K}}\right)^{2}
$$

dependence since we are dealing with a spin 2 field.

Correspondingly

$$
\Delta=\frac{i \varkappa V_{K^{\circ}} \tau_{S}}{(i \delta+I / 2) \hbar}\left(\frac{E_{K}}{M_{K}}\right)^{2}=\Delta_{0}\left(\frac{E_{K}}{M_{K}}\right)^{2}
$$

with the branching ratio, $B R$, proportional to $\Delta^{2}$. The $Y$ dependence of the $B R$ was tested several years ago with the result ${ }^{(48)} \mathrm{BR} \propto \mathrm{E}_{\mathrm{K}}^{.03 \pm .08}$ clearly excluding spin $I$ and higher fields. Our precise knowledge of the phase is the new element in the picture. Because the phase of $\Delta$ is $90^{\circ}$ away from the measured phase, $\Delta$ can be, at most, only a small part of $\eta_{+-}$.

To set new limits on $x$ we factor $\eta_{+-}$into two parts, one CP violating, which we allow, for these purposes, to have a completely arbitrary phase, and $\Delta$, with its dependence on $\gamma^{2}$. Therefore, we let

$$
\eta_{+-}=A(C P \text { violating })+\Delta_{0}\left(E_{K} / M_{K}\right)^{2}
$$

with

$$
\mathrm{BR} \propto\left|\eta_{+-}\right|^{2}
$$

We have used the phase and branching ratio data given in Table IV, solved for $\Delta$, and obtain

$$
\frac{\left|\Delta_{0}\right|}{|A|}=.006+.0035
$$


and $\arg A=37^{\circ}$ (for $x$ positive). The new limits for the weak equivalence principle are, accordingly,

$$
\begin{array}{ll}
x=1.9^{+1.0}-1.4 \times 10^{-10} & \text { earth } \\
x=1.4_{-1.0}^{+.8} \times 10^{-11} & \text { solar system } \\
x \cong 2.8^{+1.6}-2.1 \times 10^{-13} & \text { galaxy, }
\end{array}
$$

where the errors are purely statistical and do not reflect, e.g., our ignorance of the precise potential in the galaxy. The best fit for $\Delta_{0}$, and correspondingly for $x$, is slightly more than $1 \sigma$ from zero. It is certainly consistent with zero and the results above must be treated as limits. The branching ratio and phase information contribute almost equally to the result.

Measurements of the branching ratio and phase at N.A.L. energies will make it possible to extend our knowledge of the weak equivalence principle by 4 to 5 orders of magnitude. It will be highly interesting to see the result. 


\section{References}

1. J. W. Cronin, Proc. of the Fourth Hawaii Topical Conference, 1971, to be published by the University of Hawaii Press.

2. K. Winter, Proc. of Amsterdam Conference, 1971.

3. Some of these are discussed later in the context of Table IV.

4. Proc. Fourteenth International Conference on High Energy Physics, Vienna, 1968.

5. D. Jensen, Ph.D. Thesis, University of Chicago, 1970 (unpublished), and S. H. Aronson et al., Phys. Rev. Letters 23, 615 (1969).

6. S. H. Aronson et al., Phys. Rev. Letters 25, 1057 (1971).

7. M. Cullen et al., Phys. Letters 32B, 523 (1970).

8. R. Carnegie et al., Phys. Rev. D4, 1 (1971).

9. R. Carnegie, R. Cester, V. Fitch, M. Strovink, and I. Sulak, to be published.

10. We include $34.5^{\circ} \pm 10.0^{\circ}$, S. Bennett et al., Phys. Letters 29B, 317 (1969); 47.6 $\pm 12.0^{\circ}$, A. Böhm et al., Nucl. Phys. B9, 605 (1969); and $46.2^{\circ} \pm 7.4^{\circ}$, H. Faissner et al., Phys. Letters 30B, 204 (1969).

11. D. G. Hill et al., Phys. Rev. D4, 7 (1971).

12. D. F. Bartlett et al., Phys. Rev. Letters 21, 558 (1968).

13. M. Banner et al., Phys. Rev. 188, 2033 (1969).

14. R. Cence et al., Phys. Rev. Letters 22, 1210 (1969).

15. V. Barmin et aI., Phys. Letters 33B, 377 (1970).

16. I. A. Budagov et al., Phys. Letters 28B, 215 (1968).

17. J. C. Chollet et al., Phys. Letters 31B, ó58 (1970). Alone among 
the $2 \pi^{\circ}$ measurements this one actually measures $\left|\eta_{\infty}\right|$ compared to the regeneration amplitudes. We have inserted our own best estimate of the latter amplitude to obtain $\left|\eta_{00}\right|^{2}$.

18. H. Faissner et al., Nuovo Cimento 70, 57 (1970), and J. M. Gaillard et al., Nuovo Cimento 59A, 453 (1969). The number quoted is an average of two results obtained using different normalizations.

19. P. Darriulat et al., Amsterdam Conference, 1971.

20. B. Wolff et al., ibid.

21. T. T. Wu and C. N. Yang, Phys. Rev. Letters 13, 180 (1964).

22. T. D. Lee and C. S. Wu, Ann. Rev..Nucl. Sci. 16, 533 (1966).

23. C. Baltay, Phys. Rev. Letters 27, 1678 (1971).

24. R. Morse et al., Phys. Rev. Ietters 28, 388 (1972).

25. R. Abbud et al., Phys. Rev. Letters 18, 980 (1967).

26. M. Paciotti, Ph.D. Thesis, University of California, Berkeley, 1969 (unpublished), also D. Dorfan et al., Phys. Rev. Letters 19, 987 (1967).

27. S. Bennett et al., Phys. Rev. Letters 19, 993 (1967) and for final result see H. Saal, Ph.D. Thesis, Columbia University, 1969 (unpublished).

28. J. Marx et al., Phys. Letters 32B, 219 (1970).

29. V. Ashford et al., Phys. Letters 38B, 47 (1972).

30. V. Fitch, V. Hepp, D. Jensen, M. Strovink, and R. Webb, to be published.

31. G. Neuhofer et al., Contribution to Amsterdam Conference on High 
Energy Physics, 1971.

32. F. Graham et al., University of Illinois preprint C00 1895-208 (1971);

See also K. Winter, Proc. of Amsterdam Conference, 1971.

33. L. Wolfenstein, Summer School Lecture, "Ettore Majorana", 1968.

34. C. Quigg and J. D. Jackson, UCRL-18487 (1968).

35. A. Clark et al., Phys. Rev. Letters 26, 1667 (1971).

36. C. Callan and S. B. Treiman, Phys. Rev. Letters 15, 316 (1965).

37. B. Hyams et al., Phys. Letters 29B, 128 (1969).

38. N. Christ and T. D. Lee, Phys. Rev. D4, 209 (1971).

39. M. K. Gaillard, Phys. Letters 36B, 114 (1971).

40. B. Hyams et al., Phys. Letters 39B, 521 (1969).

41. D. Cline et al., University of Wisconsin report, 1971 (unpublished).

42. J. Repellin et al., Phys. Letters 36B, 603 (1971).

43. R. Morse et al., Phys. Rev. Letters 28, 388 (1972).

44. M. I. Good, Phys. Rev. 121, 311 (1961).

45. We define weak equivalence after R. H. Dicke, The Theoretical Significance of Experimental Relativity, Gordon and Breach, publishers, p. 4.

46. It is sometimes useful to know that the difference in potential seen by the $\mathrm{K}^{\circ}$ and $\overline{\mathrm{K}}^{\circ}$ when a $\mathrm{K}_{\mathrm{L}}^{\circ}$ passes through a block of material, e.g. $\mathrm{Cu}, \mathrm{K}$ momentum $=2.6 \mathrm{GeV} / \mathrm{c}$, is about $10^{-8} \mathrm{eV}$.

47. These considerations were stimulated by correspondence with $W$. Thirring some years ago. We thank Dr. Thirring for emphasizing these points about the weak equivalence principle.

48. V. Fitch, R. Roth, J. Russ, and W. Vernon, Phys. Rev. 164, 1711 (1967). 
49. J. H. Christenson et al., Phys. Rev. Letters 13, 138 (1964).

50. W. Galbraith et al., Phys. Rev. Letters 14, 383 (1965).

51. M. Bott-Bodenhausen et al., Phys. Letters $\underline{23}, 277$ (1966).

52. X. DeBouard et al., Phys. Letters 15, 58 (1965).

. 53. W. Ford et al., Phys. Rev. Letters 25, 1370 (1970).

54. F. Calaprice, private communication, and F. P. Calaprice et al., Phys. Rev. 184, 1117 (1969).

55. J. Thaler, private communication.

56. J. Baird et al., Phys. Rev. 179, 1285 (1969). 


\section{Table I}

$\begin{array}{ccc}\left|\eta_{00}\right|^{2} & \text { Compendium of }\left|\eta_{\text {oo }}\right|^{2} \text { Results } \\ -2.0 \pm 7.0 & \text { Technique } & \text { Reference } \\ 5.3 \pm 1.3 & \text { S.C. } & 12 \\ 15.2 \pm 3.6 & \text { S.C. } & 13 \\ 11.8 \pm 3.4 & \text { S.C. } & 14 \\ 3.7 \pm 1.7 & \text { HLBC } & 18 \\ 4.3 \pm 0.9 & \text { Xenon B.C. } & 16 \\ 10.0 \pm 4.2 & \text { S.C. } & 17 \\ 8.6 \pm 2.2 & \text { S.C. } & 20 \\ 3.76 \pm 0.45 & \text { S.C. + Pb GIass } & 19\end{array}$


Table II

Experimental Results on the $\mathrm{K}_{\ell}^{\circ} \rightarrow \pi^{+} \ell^{ \pm} \nu$ Charge Asymmetry
$A_{e} \times 10^{3}$
$A_{\mu} \times 10^{3}$
Ref.
$4.90 \pm 1.6$ Dorfan, et al.
26
$2.46 \pm 0.59$
Bennett et al.
27
$3.46 \pm 0.33$
Marx
28
$3.6 \pm 1.8$
Ashford
29
$2.48 \pm 0.28$
Princeton
30 


\section{TABIE III}

$\because$ Contributions to Imag $\Gamma_{12}$ from Various Decay Channels

Channel

Value $\times 10^{3}$

$K_{e 3}$
$K_{\mu 3}$
$K_{\pi^{+} \pi^{-} \pi^{\circ}}$
$K_{3 \pi^{\circ}}$

2 Imag $x\left(\right.$ Rate $K_{L} \rightarrow \pi e v /$ Rate $K_{S}$ - total) $.013 \pm .026$

2 Imag $x^{\prime}$ (Rate $K_{L} \rightarrow \pi \mu \nu /$ Rate $K_{S} \rightarrow$ total) $\quad .010 \pm .13$

Imag $\eta_{t-0}\left(\right.$ Rate $\mathrm{K}_{\mathrm{L}} \rightarrow \pi^{+} \pi^{-} \pi^{0}$ /Rate $\mathrm{K}_{\mathrm{S}} \rightarrow$ total) $.03 \pm .04$

Imag $\eta_{000}\left(\right.$ Rate $\mathrm{K}_{\mathrm{L}} \rightarrow 3 \pi^{\circ} /$ Rate $\mathrm{K}_{\mathrm{S}} \rightarrow$ total) $.05 \pm .07$

To be compared with

$\varepsilon$

$1.99 \pm .07$ 
Table IV

Predictions of Some Models of PC Violation (from Wolienstein, Ref. 33) and the Current Experimental Situation

\begin{tabular}{|c|c|c|c|c|c|c|c|c|c|}
\hline Model & $\left|\frac{n_{0}}{n_{+-}}\right|$ & $\varphi_{\varepsilon}$ & $\begin{array}{l}K^{0} \rightarrow 3 \pi \\
\text { Imag } \eta_{+-0}\end{array}$ & $\underset{\Delta}{\stackrel{ \pm}{ \pm} \rightarrow 3 \pi}$ & $\begin{array}{l}\beta \text {-decay } \\
\varphi\left(G_{A} / G_{V}\right)\end{array}$ & $\begin{array}{l}K^{\circ} \rightarrow \pi \ell \nu \\
\operatorname{Im} x\end{array}$ & $\begin{array}{l}\eta_{\rightarrow \rightarrow \pi^{+}}^{+} \pi^{-} \pi^{\circ} \\
\text { asym. }\end{array}$ & $\begin{array}{c}\mathrm{K}^{\mathrm{O}} \rightarrow \gamma+\gamma \\
\mathrm{V}_{\mathrm{a}}\end{array}$ & $\begin{array}{l}\text { EI of neut, } \\
\operatorname{Ex}\left(10^{6} \text { to } 10^{7}\right)\end{array}$ \\
\hline Strong $\overrightarrow{\Delta I}=0$ & $\simeq 1$ & $43 \pm 1$ & $\sim 10^{-3}$ & $\lesssim 10^{-3}$ & $\sim 10^{-3}$ & 0 & $<10^{-3}$ & $<10^{-2}$ & $\sim 10^{-3}$ \\
\hline Strong $\overrightarrow{\Delta I} \neq 0$ & $\neq 1$ & $43 \pm 1$ & $\sim 10^{-3}$ & $\lesssim 10^{-3}$ & $\sim 10^{-3}$ & 0 & $10^{-3}$ to $10^{-1}$ & $<10^{-2}$ & $\sim 10^{-3}$ \\
\hline Electromagnetic & $\neq 1$ & $43 \pm 1$ & $\sim 10^{-3}$ & $\lesssim 10^{-3}$ & $\sim 10^{-3}$ & 0 & $10^{-3}$ to $10^{-1}$ & large & $\sim 1$ \\
\hline Glashow $(D=0)$ & $\neq 1$ & $43 \pm 1$ & $\sim 10^{-3}$ & 0 & $10^{-3}$ & 0 & 0 & $<10^{-2}$ & $\sim 10^{-3}$ \\
\hline Glashow $(S=0)$ & 1 & 35 to 51 & .02 to .5 & .002 to .05 & $10^{-2}$ to $10^{-3}$ & 0 & 0 & $<10^{-2}$ & $\sim 10^{-2}$ to $10^{-3}$ \\
\hline$\Delta I=\frac{5}{2}$ & $\approx 2$ & a & $\sim 10^{-4}$ & $\lesssim 10^{-3}$ & 0 & 0 & 0 & $<10^{-2}$ & $<10^{-6}$ \\
\hline Sachs & 1 & $\begin{array}{l}>25 \\
<43\end{array}$ & $\eta_{+-}$ & 0 & 0 & $\neq 0$ & 0 & $r \eta_{+-}^{b}$ & $<10^{-12}$ \\
\hline Weak + e.m. & $\neq 1$ & $43 \pm 1$ & $\sim 10^{-3}$ & $\lesssim 10^{-3}$ & $\sim 10^{-3}$ & 0 & 0 & large & $\sim 1$ \\
\hline Okubo & $\neq 1$ & $43 \pm 1$ & $\sim 10^{-3}$ & $\lesssim 10^{-3}$ & $\sim 10^{-3}$ & 0 & 0 & $<10^{-2}$ & $\sim 10^{-3}$ \\
\hline Superweak & 1 & 43 & $\eta_{+-}$ & 0 & 0 & 0 & 0 & $r \eta_{+-}^{b}$ & $<10^{-8}$ \\
\hline Experiment & $1.07 \pm .08$ & $43, \pm 3$ & $-.05 \pm .24$ & $\begin{array}{c}(0.4 \pm 0.6) \\
x 10^{-3} \\
\text { and } \\
1.7 \pm 2 \times 10^{-3}\end{array}$ & .005 & $-.01 \pm .02$ & $3 \pm 20 \times 10^{-4}$ & & $\lesssim 10^{-1}$ to $10^{-2}$ \\
\hline Ref to Exp. & & & (2) & (53) & $(54)$ & (I) & (55) & & $(56)$ \\
\hline a $|\epsilon|=0, \varphi_{\epsilon}$ is & undetermi & ined & & & & & & & \\
\hline $\mathrm{b}=\frac{\gamma_{\mathrm{S}}(\gamma \gamma)}{\gamma_{\mathrm{L}}(\gamma \gamma)}+$ & $\frac{\gamma_{L}(\gamma Y)}{Y_{S}(Y Y)}$ & & & & & & & & \\
\hline
\end{tabular}




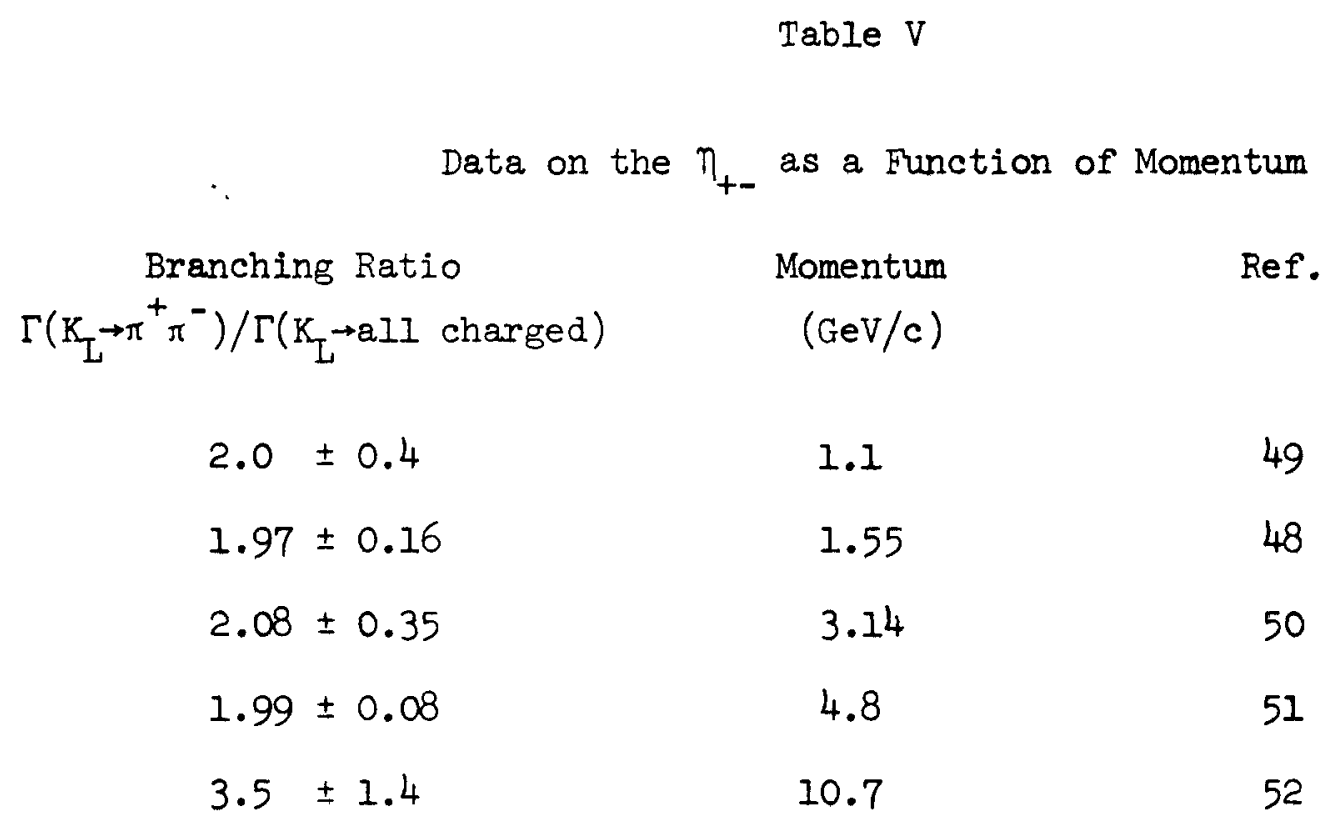

Phase

$\begin{array}{lcc}36.2^{\circ} \pm 6.1^{\circ} & 2.6 & 9 \\ 43.0^{\circ} \pm 4.0^{\circ} & 2.5 & 5 \\ 47.0^{\circ} \pm 12.0^{\circ} & 6.5 & 10 \text { (Bohm, et al.) }\end{array}$


Figure Captions

Fig. 1 The distribution of $\mathrm{K}_{\mathrm{e} 3}$ decay events with (b) and without (a) the $\mathrm{Cu}$ regenerator as a function of the separation parameter $\Delta_{\nu}=p_{\nu}\left(c \cdot m_{0}\right)-p_{\nu \perp}$. The diffractively scattered events are clearly evident in the region of negative $\Delta_{\nu}$.

Fig. 2 The charge asymmetry as a function of proper time for various regions of $\Delta_{\nu}$. The best fits, which include the diffractive and incoherent contributions, are shown.

Fig. 3 The history of the measurements of $\left|\eta_{00}\right|^{2}$. 


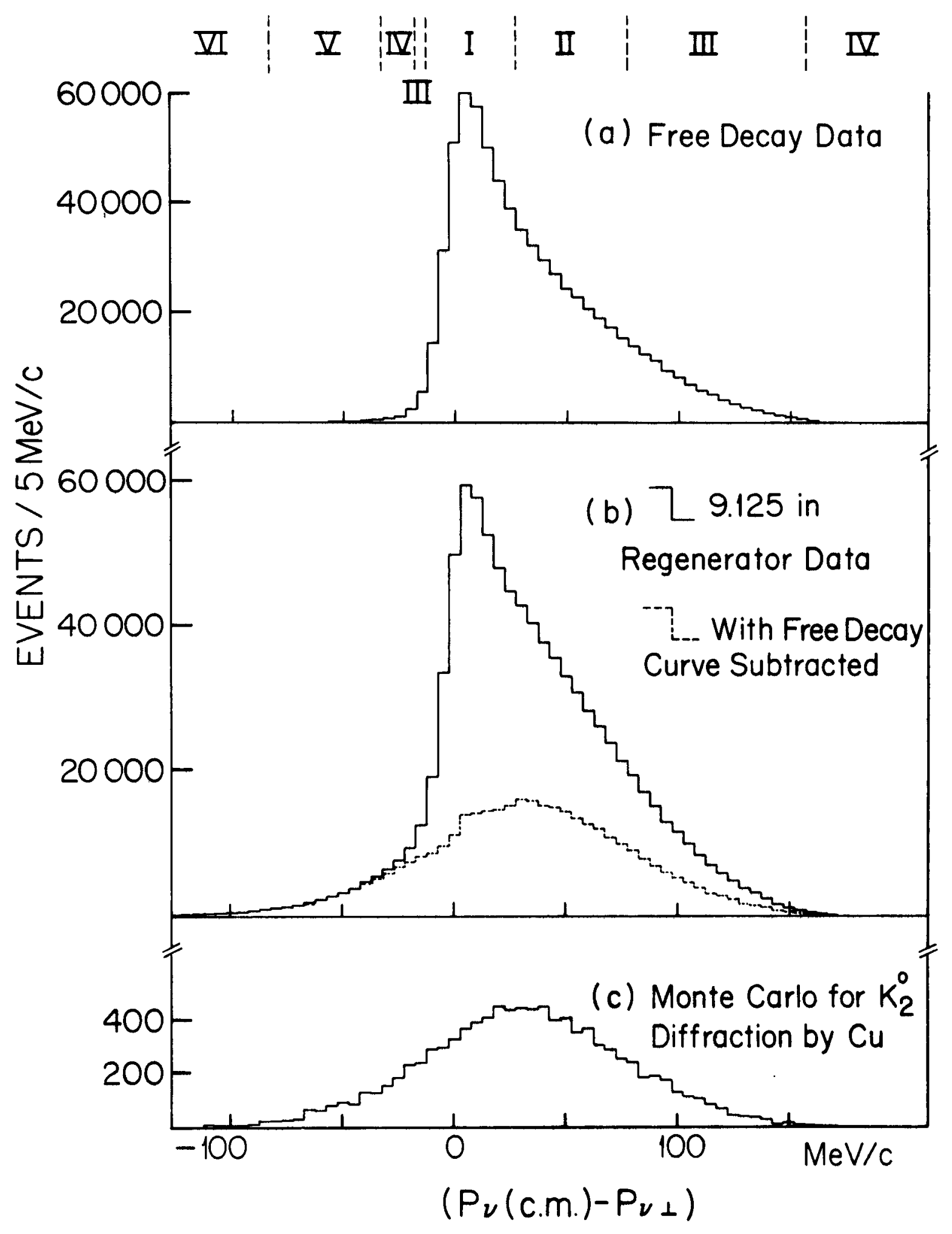




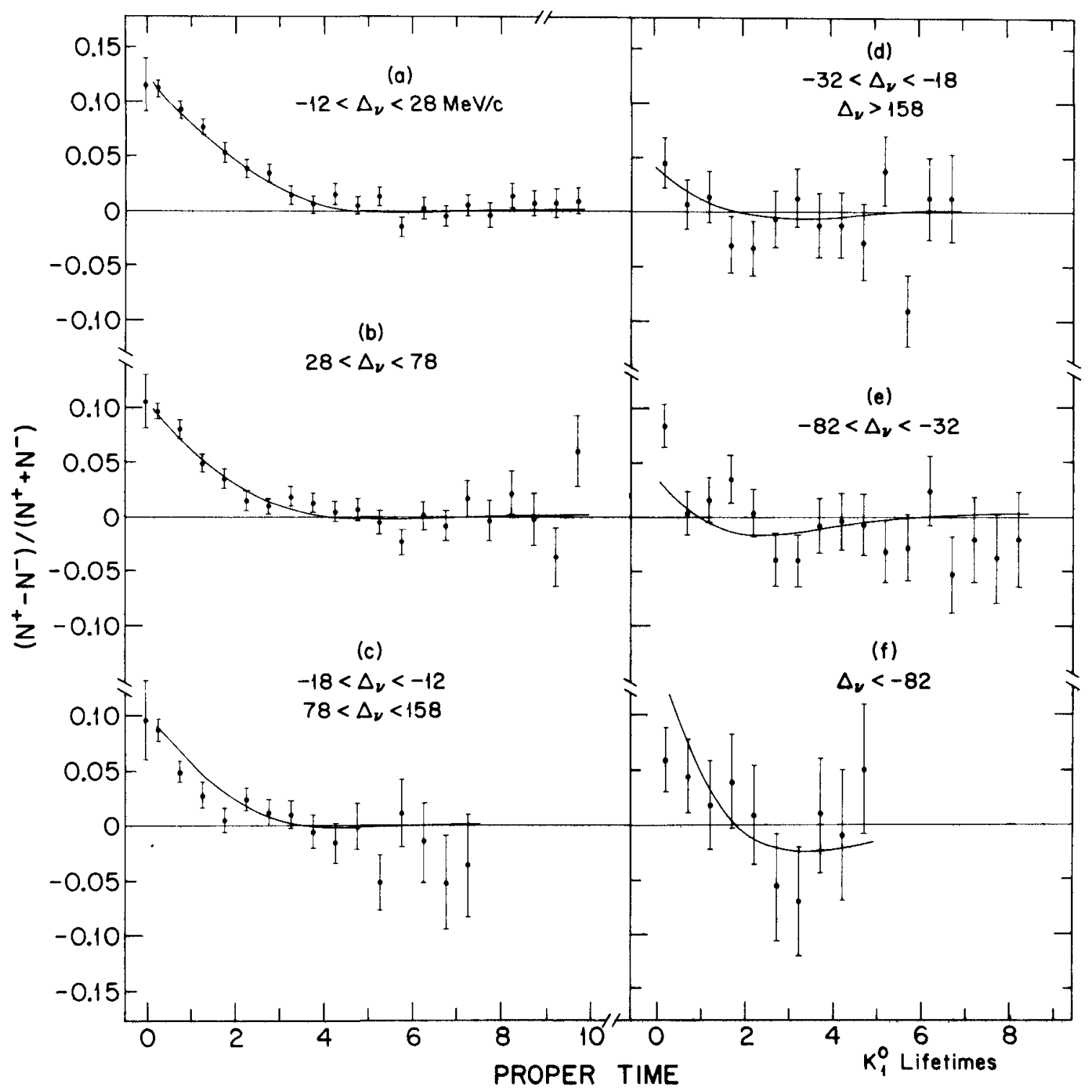

Figure 2 


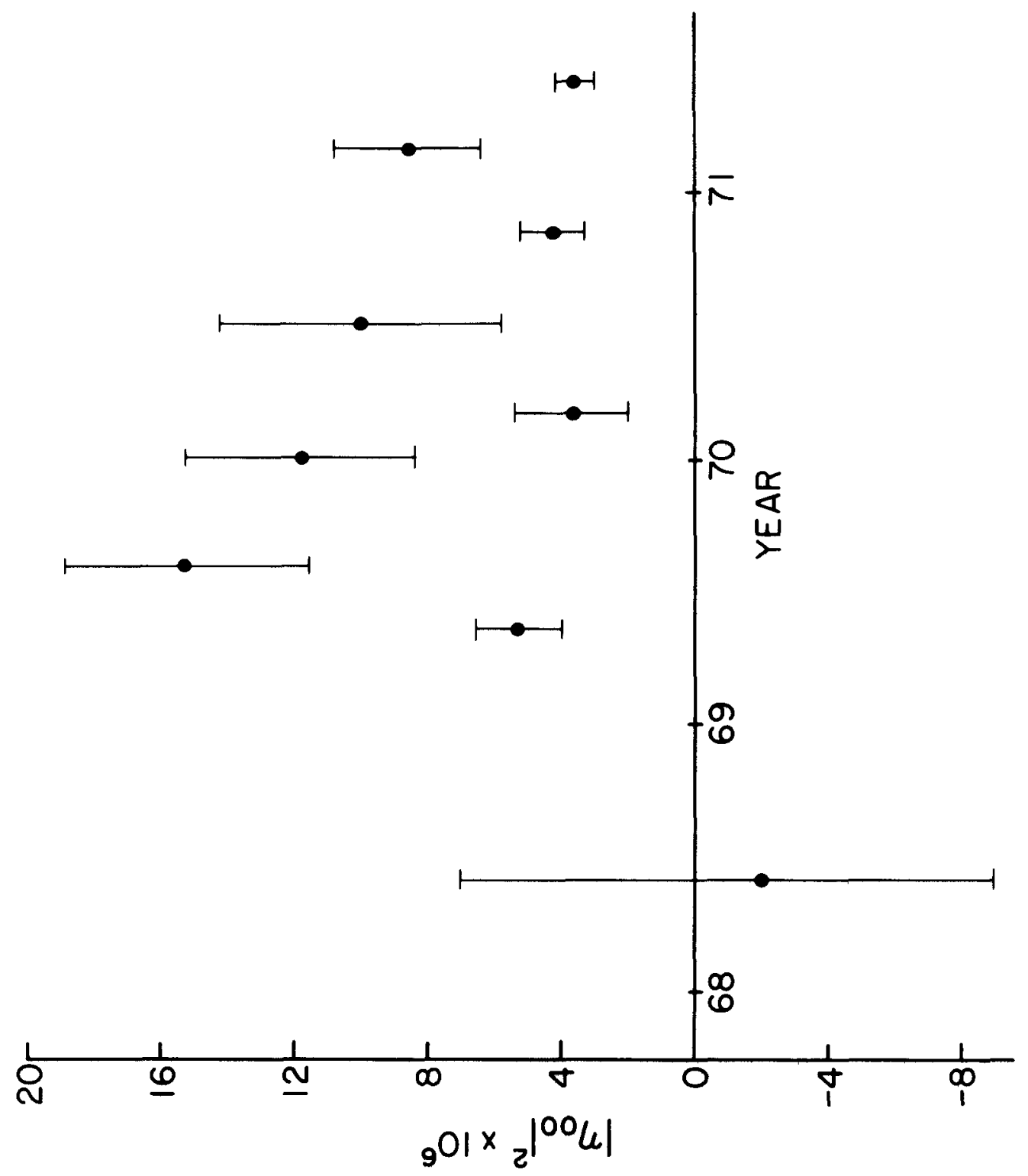

Figure 3 